\title{
Coplanar Waveguide-Fed Broadband Microwave Devices with (or without) a Thin Dielectric Substrate for Use in Flexible Electronic Systems
}

\author{
Rafal Lech, Wojciech Marynowski, Adam Kusiek, and Jerzy Mazur \\ Department of Microwave and Antenna Engineering, Gdansk University of Technology, Narutowicza 11/12, 80-233 Gdansk, Poland \\ Correspondence should be addressed to Rafal Lech; rafal.lech@eti.pg.gda.pl
}

Received 7 October 2013; Revised 22 November 2013; Accepted 1 December 2013; Published 6 January 2014

Academic Editor: Giuseppe Mazzarella

Copyright (c) 2014 Rafal Lech et al. This is an open access article distributed under the Creative Commons Attribution License, which permits unrestricted use, distribution, and reproduction in any medium, provided the original work is properly cited.

Two examples of microwave devices, fed by a coplanar waveguide and realized on a thin substrate (or without such a substrate), are employed to investigate the influence of devices' curvatures and the proximity of different materials on their parameters. To perform the tests, a broadband antenna and a low-pass filter are chosen. A feeding coplanar waveguide is realized on a dielectric material brick attached to an SMA connector and the main device structure is placed in the air or on a thin substrate. The utilization of a thin substrate or its removal from the structure gives rise to the possibility of placing the devices on curved surfaces. The investigated devices are redesigned and manufactured. The antenna has a total size of $46 \mathrm{~mm} \times 44 \mathrm{~mm}$ and covers a frequency range of $2.4-$ $35 \mathrm{GHz}$ which gives a $174 \%$ fractional bandwidth. The filter has a total size of $50 \mathrm{~mm} \times 80 \mathrm{~mm}$ and its bandwidth has a cutoff frequency of $3.4 \mathrm{GHz}$. The obtained results are verified by measurements and good agreement is achieved.

\section{Introduction}

The microwave components, including antennas and filters, used in mobile devices should not only operate within a specific frequency range but also have a compact and simple structure, with slight dimensions and a light weight. Besides the above requirements, their production costs, especially for large scale production, should also be as low as possible. When the device is designed on a thin and flexible layer, it can be bent and placed on curved surfaces. Conformal components (antennas especially) are becoming popular due to their many advantages and the possibilities of their application [1]. The advantages of using devices with a curved surface arise from the possibility of integrating them with the object on which they are mounted. In the case of conformal antennas, the particular advantage is the increase-relative to the planar antenna-of their visible angular range. The conformal devices find application in a variety of fields, such as airborne, space-borne, ship-borne and missile-borne radar, space vehicles, wireless communication, and sonar. Besides the conformability of the flexible devices, they can find application and are becoming popular in the field of flexible electronics $[2,3]$. The main requirements for such devices are light weight, low-cost manufacturing, ease of fabrication, and the availability of inexpensive flexible substrates (i.e., papers, textiles, and plastics). Many flexible antenna structures have been proposed in the literature (e.g., textiles with conducting threads [4] and paper-based [5], fluidic-based [6], and ink-jet printed antennas [7]).

In this paper, we investigate the modified version of two microwave devices. The first one is a broadband coplanar antenna proposed in $[8,9]$ with reduced dimensions, while the second one is a low-pass filter, the structure of which was proposed in [10]. In both cases, the modifications consider both the reduction and the absence of a dielectric substrate. The main goal of this work is to investigate the influence of devices' curvatures and the proximity of different materials on their performance. Both devices are designed to be fed by a coplanar line on a short brick of a dielectric layer, and the main body of the device is placed in free space (the dielectric substrate is removed). In order to manufacture such a structure, we utilize electrically-conductive adhesive tape. The designed devices are produced with the use of a laser structuring system. Commercial software is used to simulate 


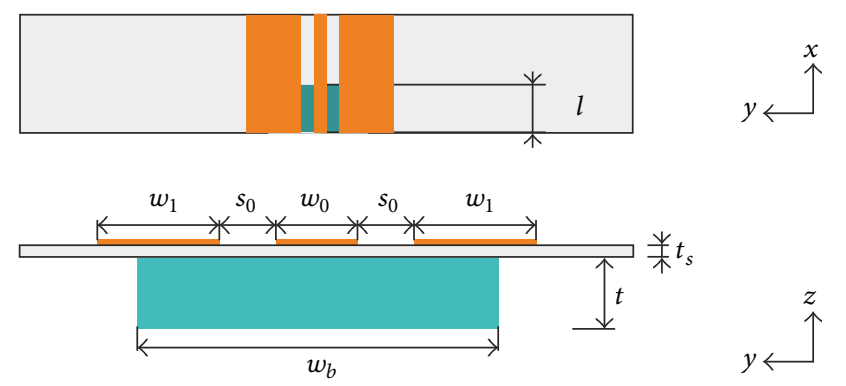

Thin substrate (optional)

Metal

Dielectric

FIGURE 1: Geometry of the feeding line.

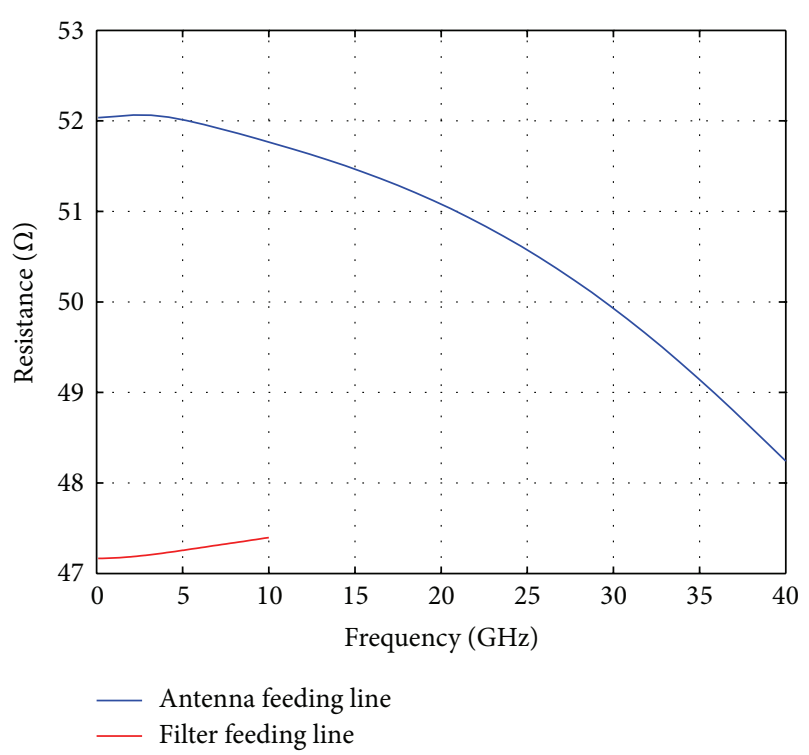

FIGURE 2: Calculated input impedance of the coplanar waveguide from Figure 1 .

the performance of the devices. The obtained results are verified by our measurements of manufactured prototypes.

\section{Geometry of the Devices}

Here, we present the schematic configurations of the investigated devices with their feeding lines and the manufacturing process used for the prototypes' realization. In Section 2.1, the description of a feeding line and a manufacturing process is presented. The antenna structure is described in Section 2.2, while the configuration of a filter is presented in Section 2.3.

2.1. The Feeding Line and Manufacturing Process. The schematic configuration of the feeding line is presented in Figure 1 . In order to provide $50 \Omega$ characteristic impedance of the coplanar waveguide for feeding the devices, the line has the dimensions $w_{0}=3.5 \mathrm{~mm}, s=0.25 \mathrm{~mm}, w_{1}=9 \mathrm{~mm}$ (in the case of the antenna structure) and $w_{1}=22 \mathrm{~mm}$ (in the case of the filter structure), and is realized on a short brick of a dielectric material with a thickness $t=1.07 \mathrm{~mm}$, a width $w_{b}=10 \mathrm{~mm}$, and a length $l=3.8 \mathrm{~mm}$, with a permittivity $\varepsilon_{r}=3.44$. The dimensions of the brick correspond to the dimensions of the SMA connector used. The calculated input impedances of these lines (for the antenna feed and the filter feed) are shown in Figure 2. In the case of the filter structure, only the frequency range from $0 \mathrm{GHz}$ to $10 \mathrm{GHz}$ is taken into consideration, while in the case of the antenna the line impedance is calculated up to $40 \mathrm{GHz}$. As can be observed, the impedance is almost frequency-invariant, and it only changes by about $3.8 \Omega$ within a frequency range of $0 \mathrm{GHz}$ to $40 \mathrm{GHz}$.

To fabricate the prototypes of the devices, we utilize an electrically-conductive adhesive tape with a copper thickness of $0.12 \mathrm{~mm}$ and a paper thickness of $t_{s}=0.08 \mathrm{~mm}$ as well as a laser structuring system. The laser cuts the structure geometry on the tape (as seen in the case of the antenna in Figure 3(a)) and after this procedure the redundant tape can be easily removed (see Figure 3(b)), resulting in the required structure geometry presented in Figure 3(c).

The dielectric brick was realized with the use of a dielectric substrate placed under the feeding line and held by the SMA connector, as shown in Figure 4.

2.2. Antenna Structure. Various designs of planar antennas have been made to increase their bandwidth $[8,9,11-17]$. The reported antenna monopoles have rectangular $[12,17]$, circular $[8,9]$, or elliptical $[11,16]$ shapes, and they can be fed by a coaxial cable or else by coplanar or microstrip lines. For these examples, a bandwidth of several gigahertz is achieved. Most designs utilize a thick, nonflexible dielectric material as an antenna substrate, which does not allow for its bending or application to curved surfaces. The coaxial cable-fed elliptical ring antenna reported in [16] was designed without the utilization of a substrate material, which allowed the obtaining of a bidirectional, symmetrical radiation pattern.

To test the influence of antenna curvature and the proximity of different materials on its performance, the antenna design proposed in [9] was employed. The schematic configuration of the antenna is presented in Figure 5. The structure is fed by a coplanar waveguide. The center strip of the line is terminated at a circular patch of radius $R_{p}$ enclosed by a circular slot of radius $R_{s}$ etched into the circular ring of a ground plane of width $R_{o}-R_{s}$. A frequency notch in the antenna can be realized by a circular slot etched into the antenna patch. The slot is of width $s_{n}$ and length $L_{n}$, with a separation width $w_{n}$ and a displacement from the patch center $d_{n}$.

As was reported in [9], the selection of a proper value of the circular patch radius $R_{p}$ ensures the acquisition of the impedance matching between the feeding line and the slot within the entire frequency range. The value of the inner slot radius $R_{s}$ influences the antenna operation only in the low frequencies. The slot offset $d_{s}$ mainly affects higher frequencies in the antenna bandwidth. The choice of the outer radius Ro of the ground plane circular ring allows for the control of the reflection characteristic slope at low 


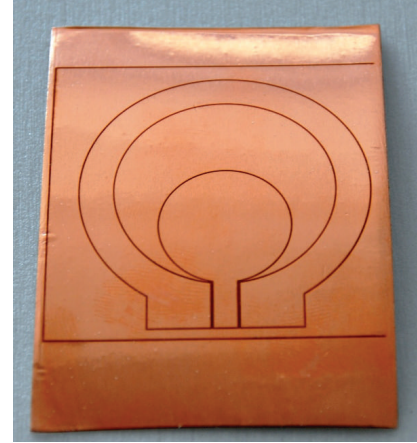

(a)

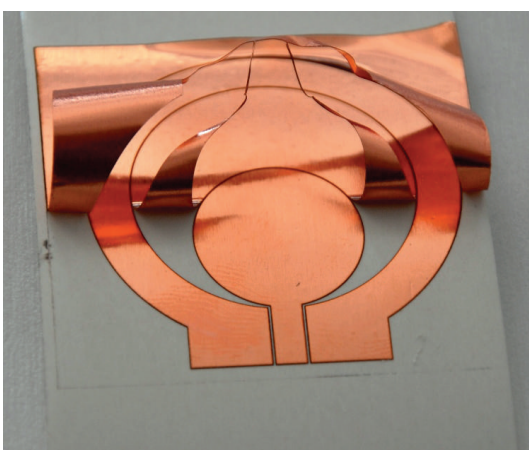

(b)

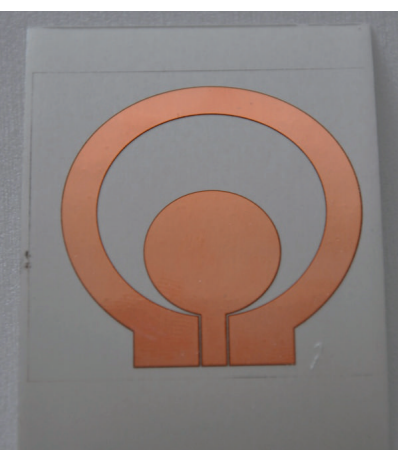

(c)

FIGURE 3: Photographs of manufactured antenna on a copper adhesive tape.

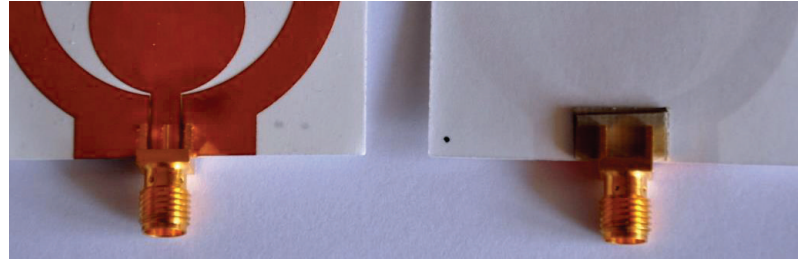

FIgure 4: Photographs of the SMA connector and the brick under the feeding line.
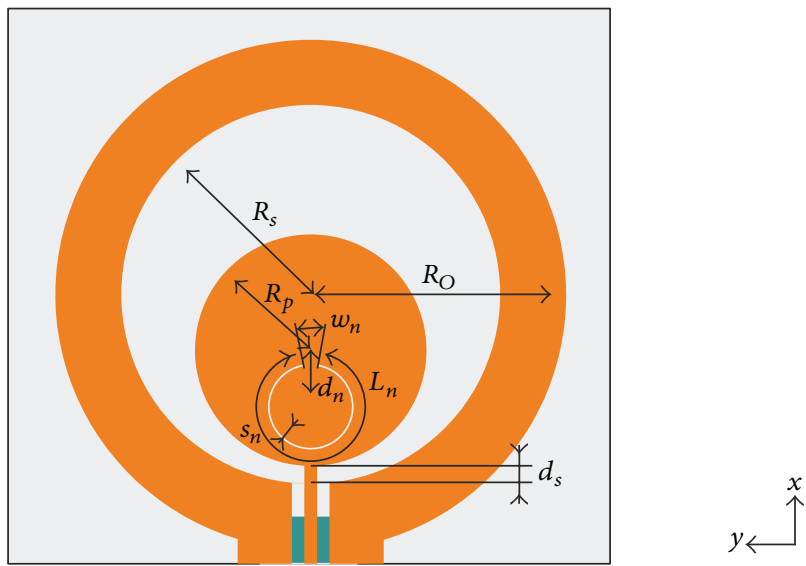

$$
\begin{aligned}
& \text { Thin substrate (optional) } \\
& \text { Metal } \\
& \text { Dielectric }
\end{aligned}
$$

FIGURE 5: Geometry of the antenna.

frequencies. The narrower the width of the ground plane circular ring, the higher the slope of the antenna reflection characteristic. To demonstrate this effect, the calculation of the slope width as a function of the ground plane circular ring width is performed and presented in the results section of this paper.

2.3. Filter Structure. The schematic configuration of the filter is presented in Figure 6. The structure consists of a transmission line with short-circuited series stubs. The stubs are of

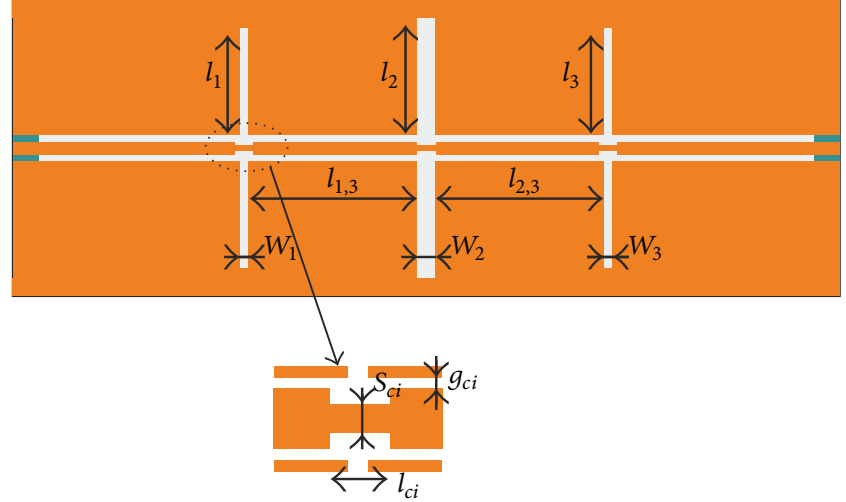

Thin substrate (optional)

Metal

Dielectric

Figure 6: Geometry of the filter.

length $l_{i}$ and width $W_{i}$ for $i=1,2,3$. The distances between the stubs are $l_{1,2}$ and $l_{2,3}$. At the connection points between the coplanar waveguide and the slot lines, the compensated crossjunction is utilized to minimize the unwanted parasitic effect. The cross-junction structure is constructed with a narrowed strip conductor with a width $s_{c i}$ and length $l_{c i}$.

\section{Results}

The scattering parameters of the manufactured devices were measured using an Anritsu/Wiltron 37269A VNA Network Analyzer, and the radiation patterns of the antenna were measured in an anechoic chamber using an Agilent E5071C ENA Network Analyzer with a GeoZondas UWB AU3.1G10.6G-1 antenna for measurements at $4 \mathrm{GHz}$ and $6 \mathrm{GHz}$ and a waveguide horn antenna EMCO Model no. 3160-07 for measurements at $10 \mathrm{GHz}$.

3.1. Antenna Structure. In order to demonstrate the accuracy of the proposed fabrication process, we designed, manufactured and measured four broadband antennas with the dimensions listed in Table 1. 

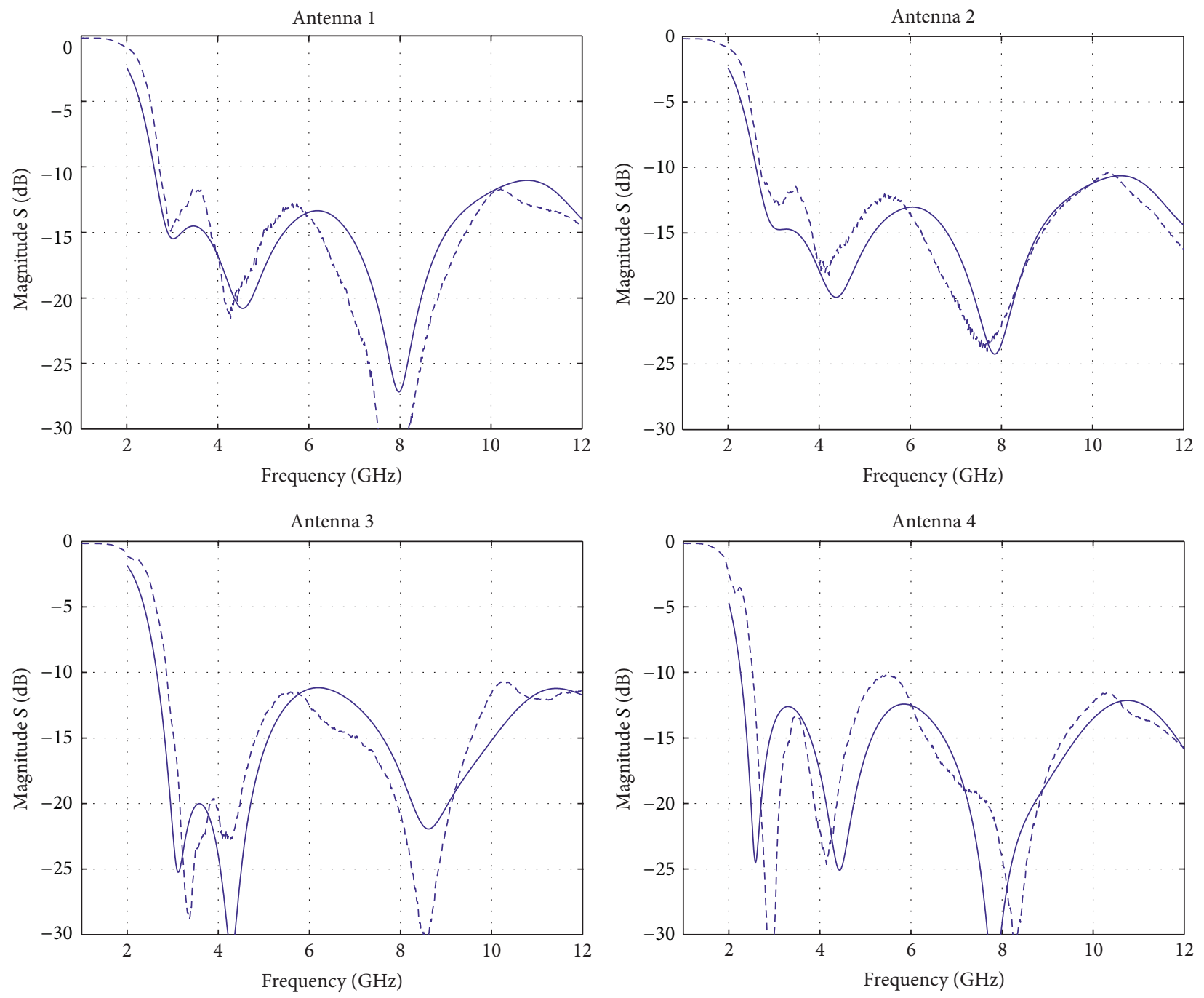

FIGURE 7: Magnitude of the reflection coefficient for planar antennas. Simulation: solid line. Measurement: dashed line.

TABLE 1: Dimensions of the designed antennas. All dimensions are in millimeters.

\begin{tabular}{lcccc}
\hline & $R_{o}$ & $R_{s}$ & $R_{p}$ & $d_{s}$ \\
\hline Antenna no. 1 & 21 & 16 & 9.5 & 0.15 \\
Antenna no. 2 & 21.5 & 16 & 9.5 & 0.2 \\
Antenna no. 3 & 21.5 & 16 & 8.5 & 0.2 \\
Antenna no. 4 & 22 & 18 & 10 & 0.1 \\
\hline
\end{tabular}

To obtain the antenna dimensions, commercial electromagnetic software was used to simulate and optimize the structure. The simulated and measured characteristics of the reflection coefficients versus frequency are illustrated in Figure 7.

As can be observed from the results, the simulated and measured characteristics for each antenna are in good agreement with each other, which validates the proposed realization technique.
For further investigation, we considered only antenna no. 4. The lack of a thick dielectric substrate below the radiator allows for the bending of the antenna, as shown in Figure 8, in order to place it on a curved surface.

First, the investigation of the reflection coefficient characteristic slope width $\Delta=f\left(\left|S_{11}\right|=-10 \mathrm{~dB}\right)-f\left(\left|S_{11}\right|=\right.$ $-3 \mathrm{~dB})$ as a function of the ground plane circular ring width $w_{g}=R_{o}-R_{s}$ is performed. The slope width $\Delta$ is defined as a difference of frequencies at which the magnitude of the reflection coefficient takes the values $-3 \mathrm{~dB}$ and $-10 \mathrm{~dB}$. The results are calculated for the planar and curved cases of the antenna and are presented in Table 2.

As can be seen, the narrower the width of the ground plane circular ring, the higher the slope of the antenna reflection characteristics. Therefore, the choice of radius $R_{o}$ can be used to adjust the slope of the reflection coefficient characteristics.

Next, the measurement of the antenna reflection coefficients for the planar and curved cases was performed, with the results presented in Figure 9. 


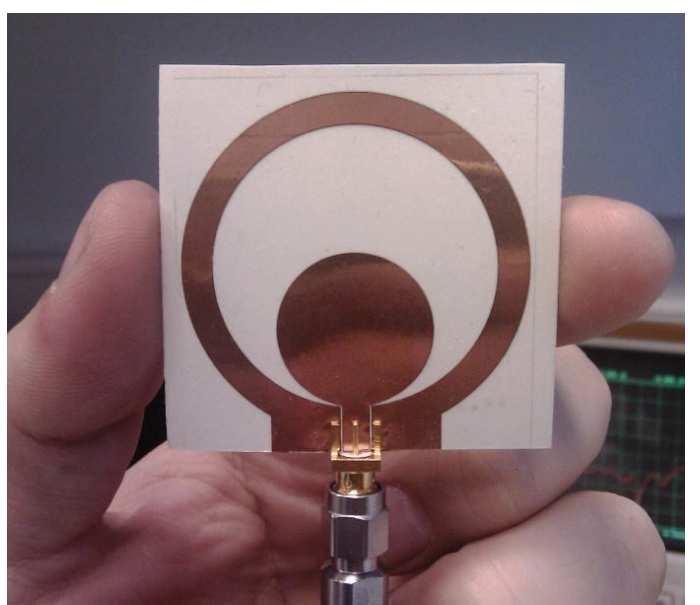

(a)

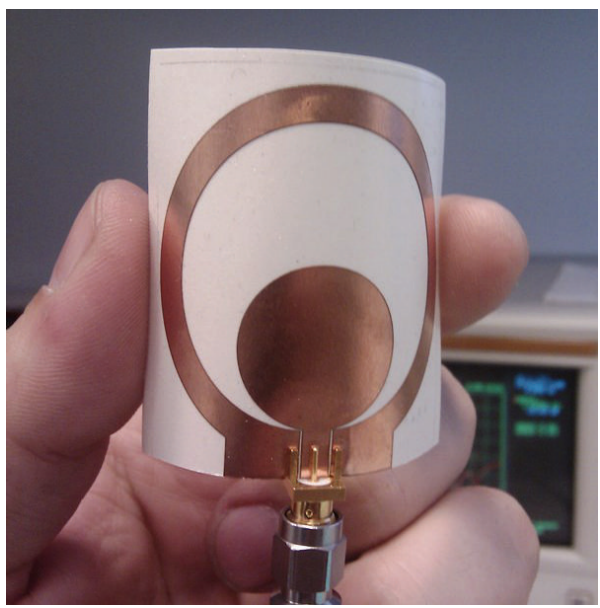

(c)

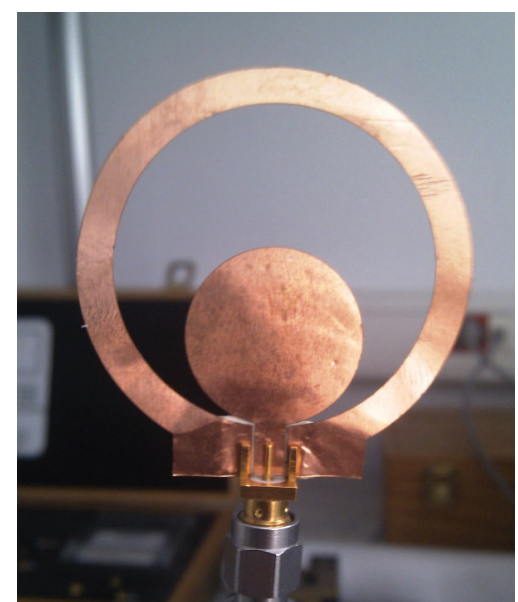

(b)

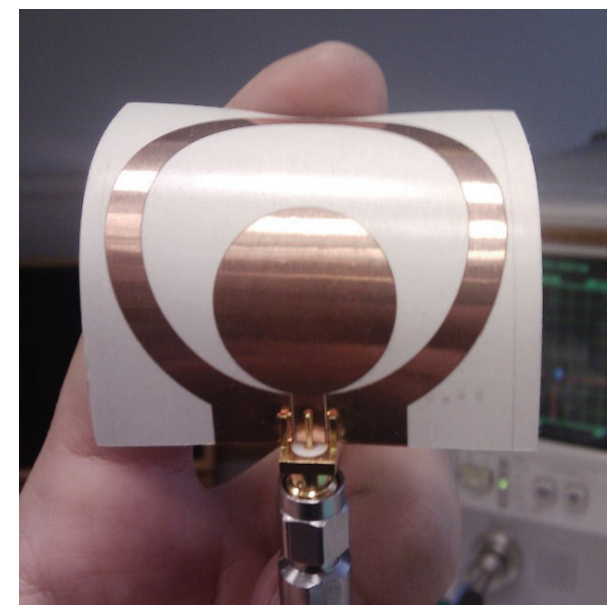

(d)

FIGURE 8: Photographs of the fabricated antenna: (a) planar version on paper, and (b) planar version in air, (c) curved around the $x$-axis, (d) curved around the $y$-axis.

TABLE 2: The slope width $\Delta(\mathrm{MHz})$ as a function of the ground plane circular ring width $w_{g}=R_{o}-R_{s}$ for antenna no. 4 . The width of the ground plane circular ring is regulated by changing the radius $R_{o}$ with the other dimensions unchanged. The curvature radii $13 \mathrm{~cm}$ and $30 \mathrm{~cm}$ were assumed for the antennas curved around the $x$-axis and the $y$-axis, respectively.

\begin{tabular}{lccccc}
\hline$w_{g}(\mathrm{~mm})$ & 1 & 4 & 7 & 10 & 12 \\
$\Delta$-planar case & 380 & 412 & 470 & 556 & 708 \\
$\Delta$-curved $x$-axis & 292 & 293 & 319 & 412 & 657 \\
$\Delta$-curved $y$-axis & 307 & 410 & 532 & 584 & 607 \\
\hline
\end{tabular}

As can be seen, the planar version of the antenna operates within a frequency range of $2.4-35 \mathrm{GHz}$ with a reflection coefficient lower than $-10 \mathrm{~dB}$ (which gives a $174 \%$ fractional bandwidth). In the case of bending the antenna around the $y$-axis, the operation frequency is limited to $30.7 \mathrm{GHz}$. Removing the paper substrate slightly deforms the antenna structure and, in this case, the upper frequency is limited to $29.5 \mathrm{GHz}$. The discrepancies between the simulated and
TABLE 3: Dimensions of the resonators realizing the frequency notch. All dimensions are in millimeters.

\begin{tabular}{lcccc}
\hline & $L_{n}$ & $s_{n}$ & $w_{n}$ & $d_{n}$ \\
\hline Notch at $5 \mathrm{GHz}$ & 13.5 & 0.3 & 0.6 & 4 \\
Notch at $6 \mathrm{GHz}$ & 11 & 0.3 & 0.6 & 5 \\
\hline
\end{tabular}

measured results at higher frequencies may be due to the SMA connector.

When realizing the antenna with a frequency notch on the adhesive tape, the parameters of the paper need to be taken into account, as they may affect the resonance of the notch. The paper is of thickness $t_{p}=0.08 \mathrm{~mm}$ and its permittivity was determined experimentally to be $\varepsilon_{r}=2.9$. Two antennas with notches at frequencies $5 \mathrm{GHz}$ and $6 \mathrm{GHz}$ were designed and manufactured. The dimensions of the resonator are presented in Table 3.

The results of the reflection coefficients for the antenna with a notch are presented in Figure 10. As can be observed, the influence of the paper is clearly visible, as the resonance 


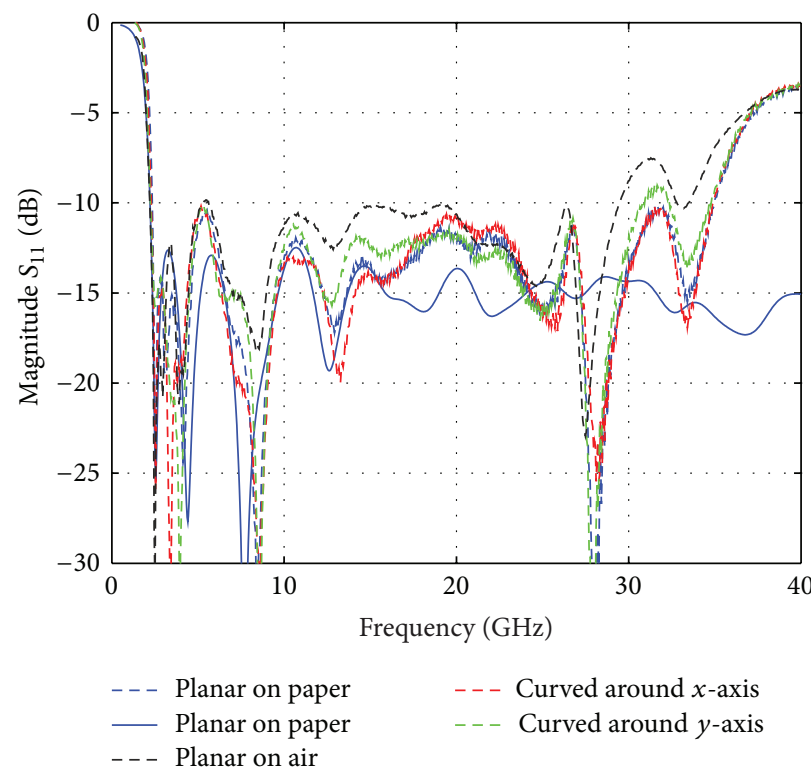

FIGURE 9: Magnitude of the reflection coefficient for the planar and curved antennas, as shown in Figure 8. Simulation: solid line. Measurement: dashed line.
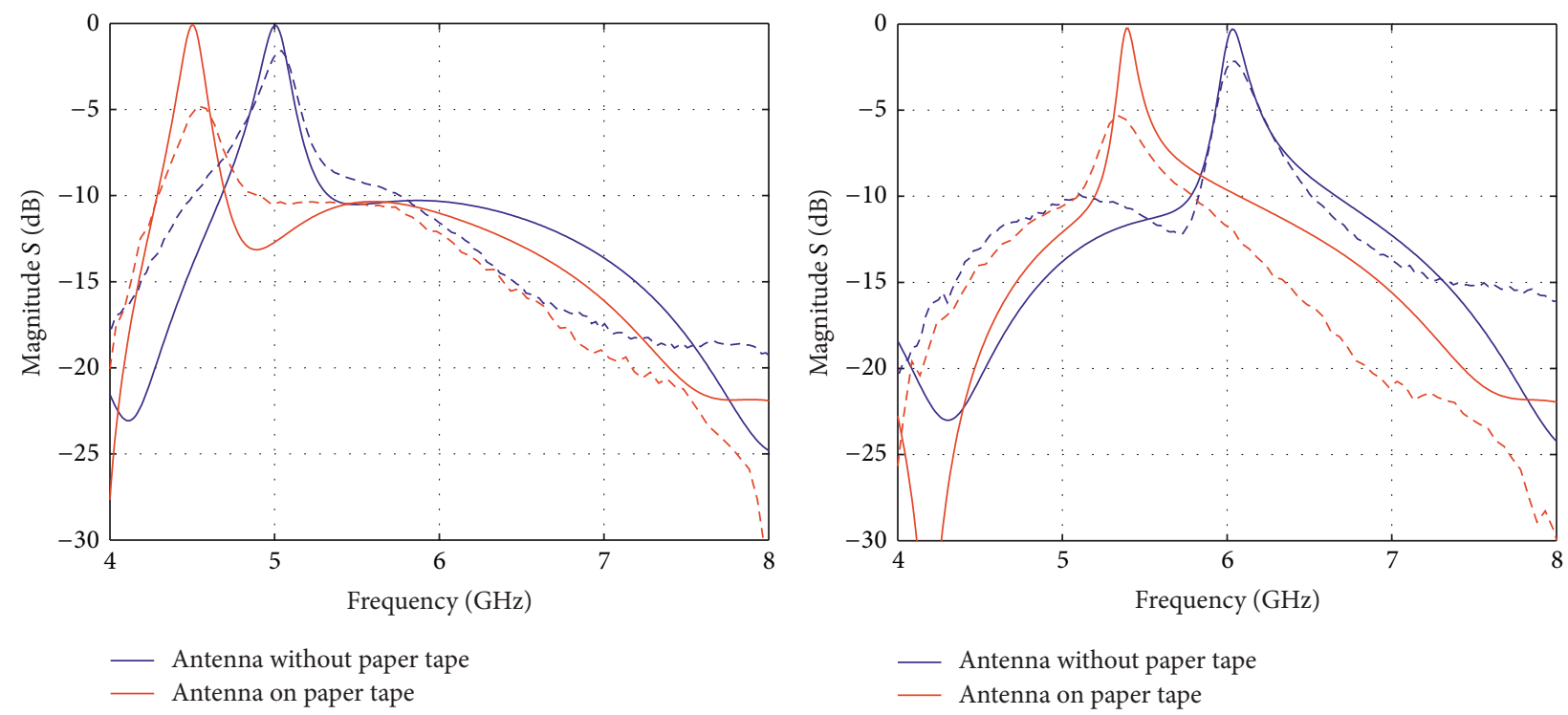

FIGURE 10: Magnitude of the reflection coefficient for a planar antenna with a notch at frequencies: (a) $f=5 \mathrm{GHz}$, (b) $f=6 \mathrm{GHz}$. Simulation: solid line. Measurement: dashed line.

of the notch is shifted in both cases by about $500 \mathrm{MHz}$ in comparison to the antennas without the paper tape.

The antenna without the notch was next attached to several different materials, such as a wooden $\operatorname{desk}\left(\varepsilon_{r} \approx\right.$ 2) of thickness $3 \mathrm{~cm}$, plastic foil $\left(\varepsilon_{r} \approx 2.5\right)$ of thickness $0.13 \mathrm{~mm}$, window glass $\left(\varepsilon_{r} \approx 4.8\right)$ of thickness $5 \mathrm{~mm}$, a human arm, and a cylindrical PCV pipe $\left(\varepsilon_{r} \approx 3\right)$ of radius $5 \mathrm{~cm}$ and thickness $2 \mathrm{~mm}$. The antenna reflection coefficient was measured within the frequency range of $0 \mathrm{GHz}$ up to $15 \mathrm{GHz}$, and the results are presented in Figure 11. As can be seen, the proximity of these materials slightly increases the antenna reflection coefficient; however, it does not significantly change the antenna performance.
Finally, the fabricated antenna was measured in an anechoic chamber at frequencies of $4 \mathrm{GHz}, 6 \mathrm{GHz}$, and $10 \mathrm{GHz}$, and the results were compared with those obtained from the simulations. The radiation patterns for the investigated antennas are presented in Figure 12. As can be seen, good agreement between the simulations and the measurements was achieved.

3.2. Filter Structure. The filter proposed in [10] was redesigned to the structure situated on the paper substrate. The obtained dimensions are as follows: $l_{1}=l_{2}=l_{3}=11.4 \mathrm{~mm}$, $W_{1}=W_{3}=0.11 \mathrm{~mm}, W_{2}=0.45 \mathrm{~mm}, l_{1,2}=l_{2,3}=22.8 \mathrm{~mm}$, 


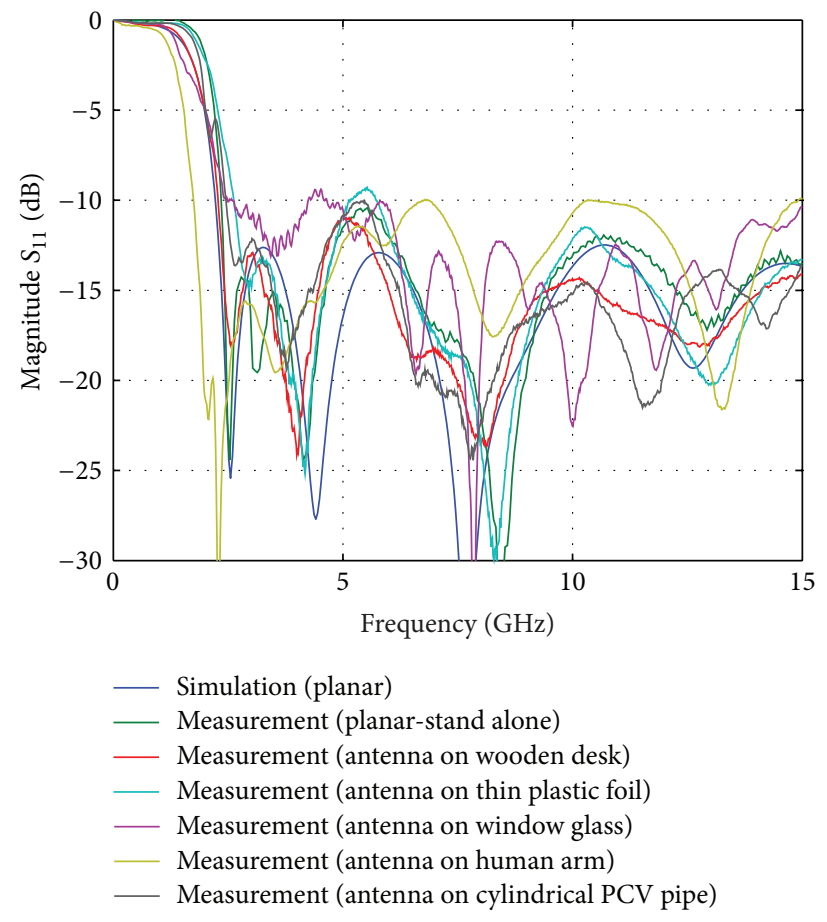

FIGURE 11: Magnitude of the reflection coefficient for the investigated antenna placed on different materials.
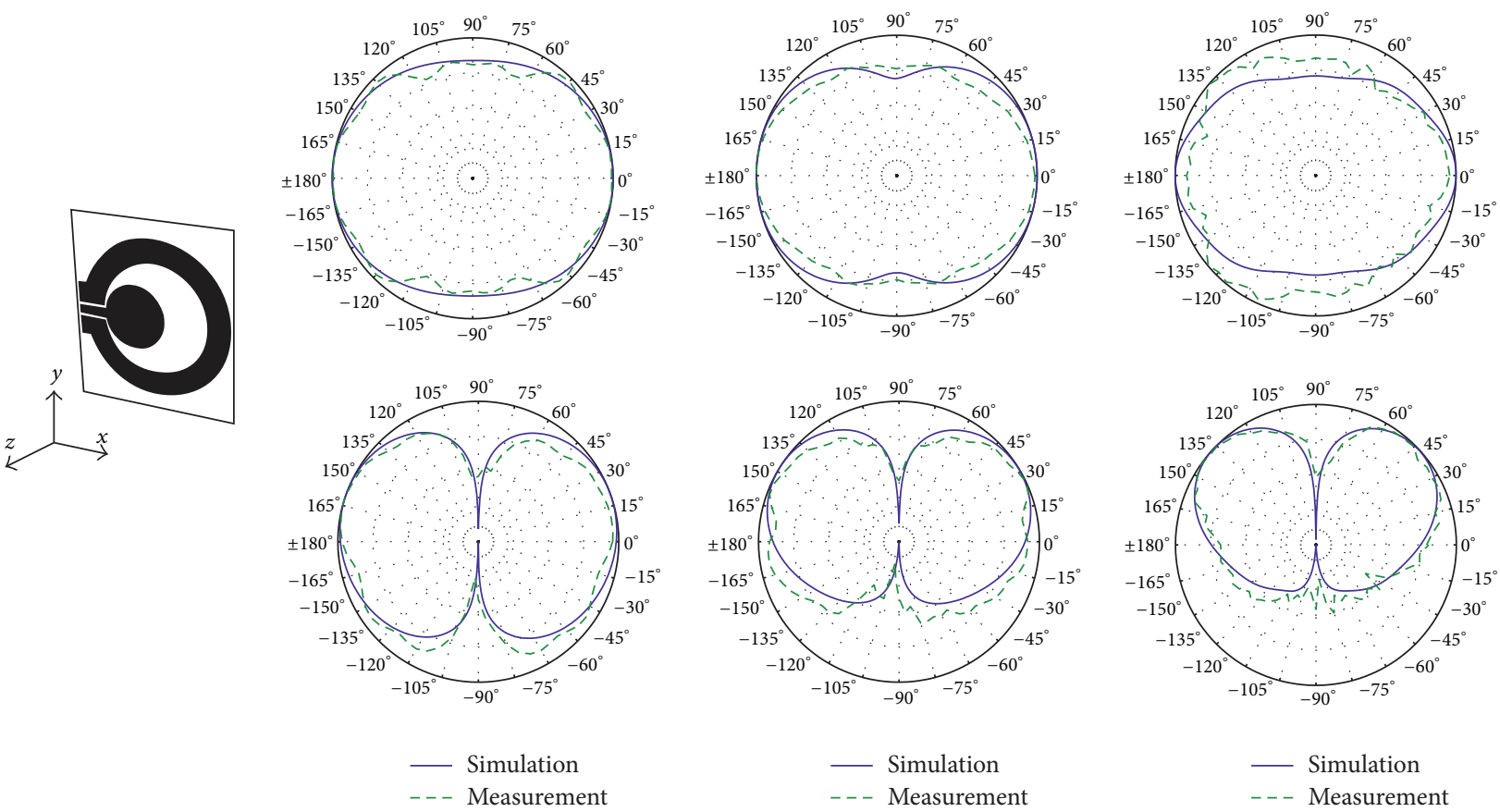

FIGURE 12: Radiation patterns of the antenna at frequencies $4 \mathrm{GHz}, 6 \mathrm{GHz}$, and $10 \mathrm{GHz}$. First column: $y z$ plane for $x=0$, second column: $x z$ plane for $y=0$. Simulation: solid line. Measurement: dashed line.

$s_{c 1}=s_{c 3}=2.28 \mathrm{~mm}, s_{c 2}=2.23 \mathrm{~mm}, l_{c 1}=l_{c 3}=0.17 \mathrm{~mm}$, and $l_{c 2}=0.33 \mathrm{~mm}$.

Also, in this case, the lack of a thick dielectric substrate below the structure allows for the bending of the filter, as shown in Figure 13.
The simulated and measured characteristics of the scattering parameters versus frequency are illustrated in Figures 14 and 15.

As can be observed from the results, bending the filter does not significantly change its scattering parameters. The 


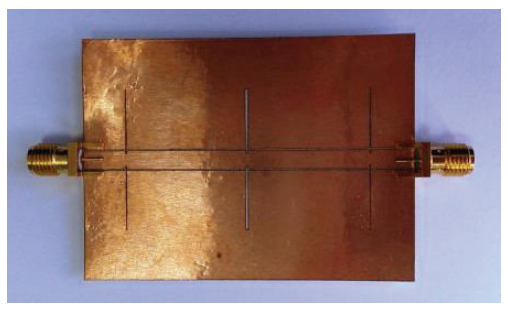

(a)

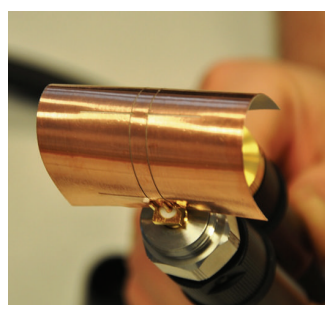

(b)

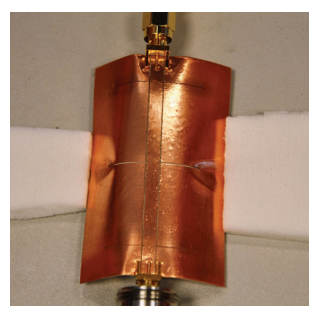

(c)

FIGURE 13: Photographs of the fabricated filter: (a) planar version, (b) curved along its length, and (c) curved along its width.

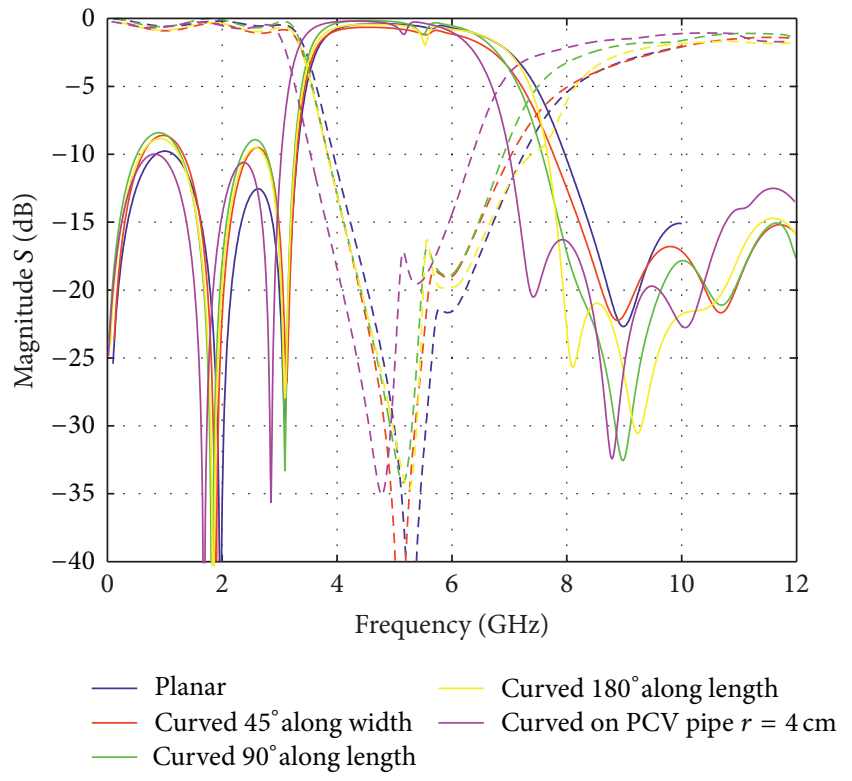

FIGURE 14: Simulated magnitude of the scattering parameters for the filter in its planar and curved version. $S_{11}$ : solid line. $S_{21}$ : dashed line.

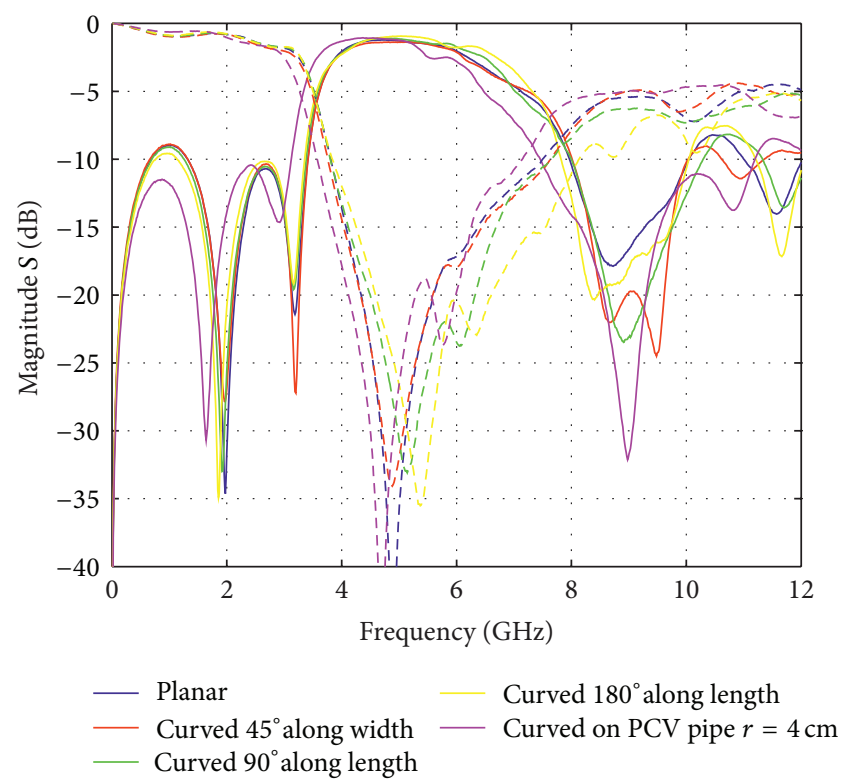

FIGURE 15: Measured magnitude of the scattering parameters for the filter in its planar and curved version. $S_{11}$ : solid line. $S_{21}$ : dashed line. proximity of other materials will affect its performance as the electric lengths of the stubs and lines are changed. Placing the filter on the PCV pipe ( $\varepsilon_{r} \approx 3$, radius $5 \mathrm{~cm}$, thickness $2 \mathrm{~mm}$ ) lowered its cutoff frequency by about $270 \mathrm{MHz}$.

\section{Conclusion}

The investigation of the influence of microwave devices' curvatures and the proximity of different materials on their performance was performed. Two examples of microwave devices, a broadband antenna and a low-pass filter, were employed for the investigation. The devices were realized on adhesive copper tape. A lack of a dielectric substrate allowed for the bending of the devices so as to place them on curved surfaces. The bending of the antennas and the proximity of several different materials do not significantly affect their performance. In the case of the filter structure, the bending does not affect its performance but the proximity of other materials changes the electrical lengths of the series stubs and lines, which has an influence on the filter parameters. In summary, the greater the broadband character of the device, the less sensitive it is to the proximity of other materials. In addition, as the device curvature or the other material's proximity may increase the reflection coefficient of the device, this effect should be taken into account in the design process of the basic structure by setting slightly rigorous design goals. The conducted investigations confirm that the proposed antenna and filter structures can be realized on thin (or without) substrates and that they can be utilized in flexible electronic systems.

\section{Conflict of Interests}

The authors declare that there is no conflict of interests regarding the publication of this paper.

\section{Acknowledgments}

This work was supported in part by National Science Center under Grant decision no. DEC-2011/01/D/ST7/06639 and by Polish Ministry of Science and Higher Education from sources for science in the years 2012-2013 under Contract no. 0340/IP2/2011/71. 


\section{References}

[1] L. Josefsson and P. Persson, "Conformal array synthesis including mutual coupling," Electronics Letters, vol. 35, no. 8, pp. 625627, 1999.

[2] A. Nathan and B. R. Chalamala, "Special issue on flexible electronics technology, part 1: systems and applications," Proceedings of the IEEE, vol. 93, no. 7, pp. 1235-1237, 2005.

[3] J. Hu, "Overview of flexible electronics from ITRI's viewpoint," in Proceedings of the 28th VLSI Test Symposium (VTS '10), p. 84, Santa Cruz, Calif, USA, April 2010.

[4] S. Zhang, A. Chauraya, W. Whittow et al., "Embroidered wearable antennas using conductive threads with different stitch spacings," in Proceedings of the 7th Loughborough Antennas and Propagation Conference (LAPC '12), pp. 1-4, Loughborough, UK, November 2012.

[5] D. E. Anagnostou, A. A. Gheethan, A. K. Amert, and K. W. Whites, "A direct-write printed antenna on paper-based organic substrate for flexible displays and WLAN applications," Journal of Display Technology, vol. 6, no. 11, pp. 558-564, 2010.

[6] M. Kubo, X. Li, C. Kim et al., "Stretchable microfluidic radiofrequency antennas," Advanced Materials, vol. 22, no. 25, pp. 27492752, 2010.

[7] H. R. Khaleel, H. M. Al-Rizzo, and A. I. Abbosh, "Design, fabrication, and testing of flexible antennas," in Advancement in Microstrip Antennas With Recent Applications, A. Kishk, Ed., InTech, Vienna, Austria, 2013.

[8] T. A. Denidni and M. A. Habib, "Broadband printed CPW-fed circular slot antenna," Electronics Letters, vol. 42, no. 3, pp. 135136, 2006.

[9] W. Marynowski and J. Mazur, "Design of UWB coplanar antenna with reduced ground plane," Journal of Electromagnetic Waves and Applications, vol. 23, no. 13, pp. 1707-1713, 2009.

[10] R. Li, S. Sun, and L. Zhu, "Direct synthesis of transmission line low-/high-pass filters with series stubs," IET Microwaves, Antennas \& Propagation, vol. 3, no. 4, pp. 654-662, 2009.

[11] E. S. Angelopoulos, A. Z. Anastopoulos, D. I. Kaklamani, A. A. Alexandridis, F. Lazarakis, and K. Dangakis, "Circular and elliptical CPW-fed slot and microstrip-fed antennas for ultrawideband applications," IEEE Antennas and Wireless Propagation Letters, vol. 5, no. 1, pp. 294-297, 2006.

[12] Y. F. Liu, K. L. Lau, Q. Xue, and C. H. Chan, "Experimental studies of printed wide-slot antenna for wide-band applications," IEEE Antennas and Wireless Propagation Letters, vol. 3, no. 1, pp. 273-275, 2004.

[13] M. E. Bialkowski and A. M. Abbosh, "Design of UWB planar antenna with improved cut-off at the out-of-band frequencies," IEEE Antennas and Wireless Propagation Letters, vol. 7, pp. 408410, 2008.

[14] S.-W. Su, K.-L. Wong, and F.-S. Chang, "Compact printed ultra-wideband slot antenna with a band-notched operation," Microwave and Optical Technology Letters, vol. 45, no. 2, pp. 128130, 2005.

[15] M. Chen and J. Wang, "Compact CPW-fed circular slot antenna for ultra-wideband applications," in Proceedings of the 8th International Symposium on Antennas, Propagation and EM Theory (ISAPE '08), pp. 78-81, Kunming, China, November 2008.

[16] K. Chawanonphithak, C. Phongcharoenpanich, S. Kosulvit, and M. Krairiksh, " $5.8 \mathrm{GHz}$ notched UWB bidirectional elliptical ring antenna excited by circular monopole with curved slot," in Proceedings of the Asia-Pacific Microwave Conference (APMC '07), pp. 1-4, Bangkok, Thailand, December 2007.

[17] D. Kumar, T. Singh, R. Dwivedi, and S. Verma, "A compact monopole CPW-fed dual band notched square-ring antenna for UWB applications," in Proceedings of the 4th International Conference on Computational Intelligence and Communication Networks (CICN '12), pp. 57-60, Mathura, India, November 2012. 

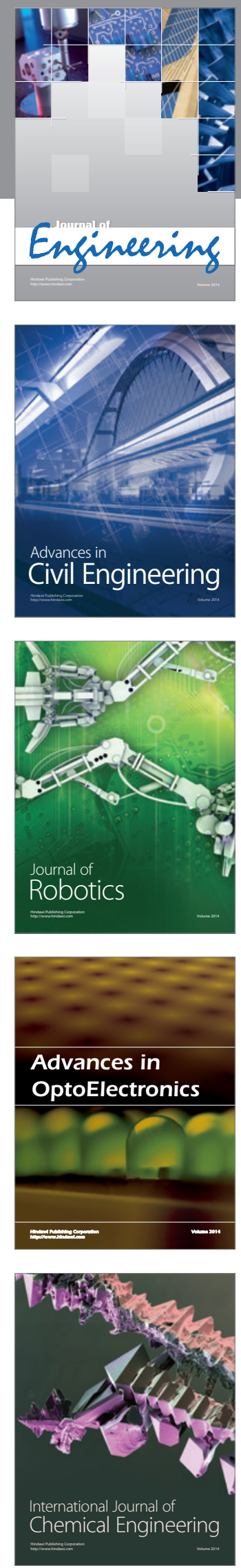

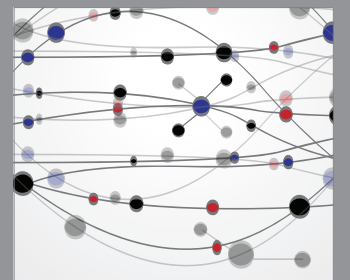

The Scientific World Journal
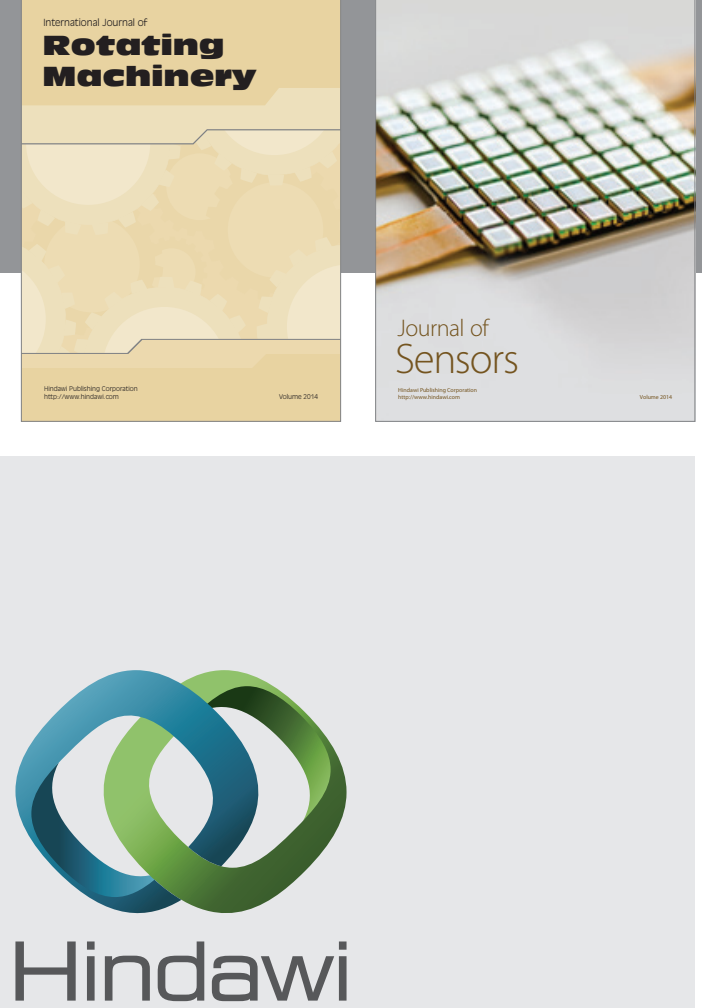

Submit your manuscripts at http://www.hindawi.com
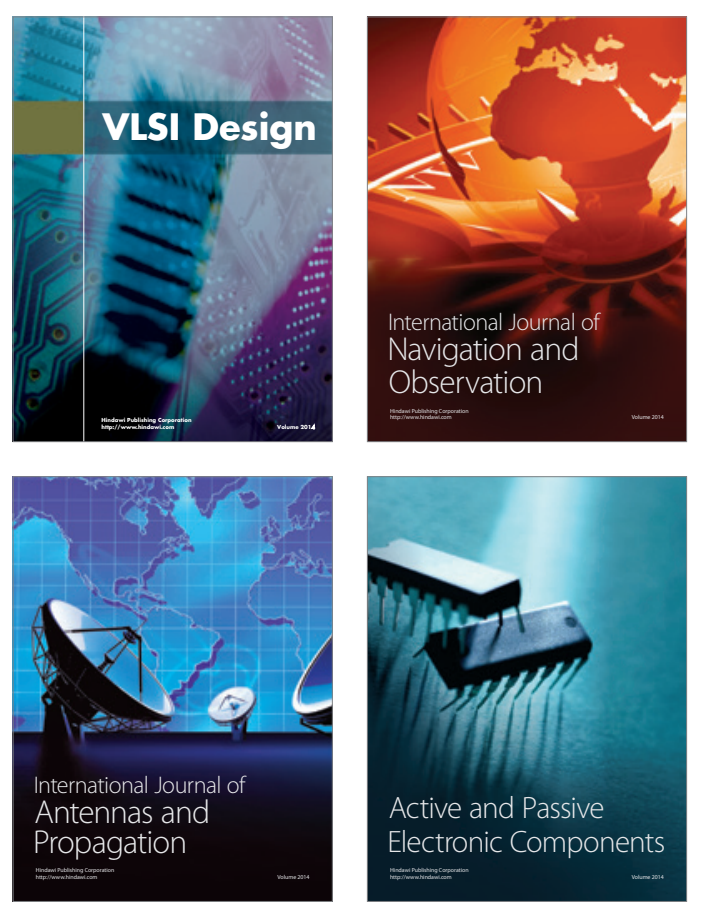
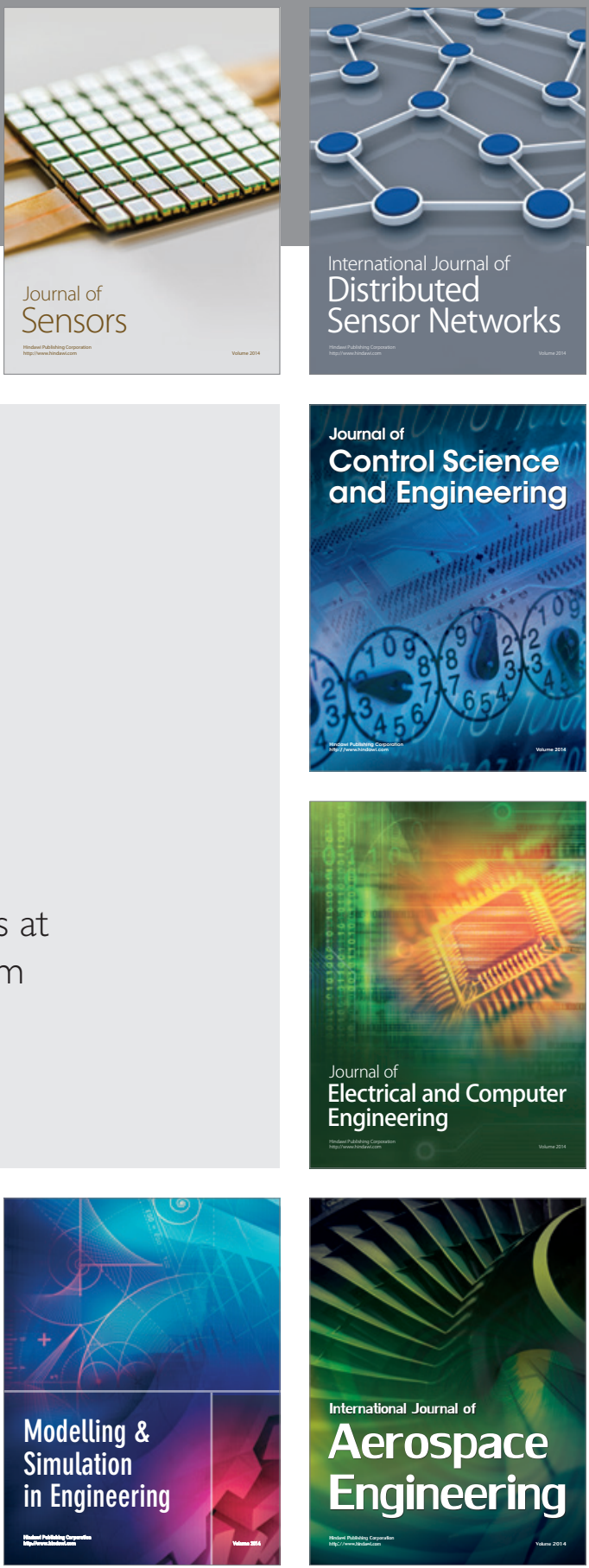

Journal of

Control Science

and Engineering
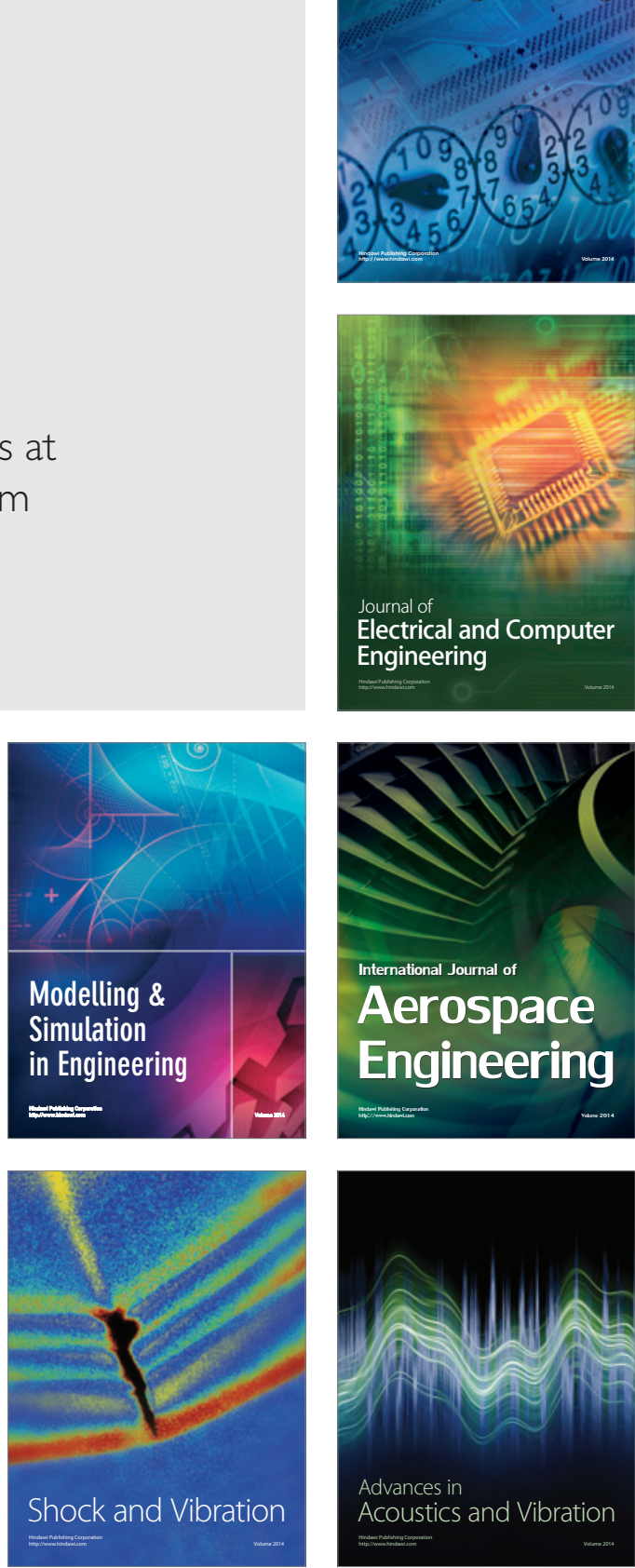